\title{
Biomechanics Analysis of Side Header in Soccer Players
}

\author{
Fuming $\mathrm{Gu}^{1, \text { a }}$ \\ ${ }^{1}$ Department of Sports Medicine, Chengdu Sport University, Chengdu 610041, China; \\ a1165037369@qq.com
}

Keywords: Kinetics, header, soccer

\begin{abstract}
The paper analyzed the side header in soccer using kinetics The finding indicated that (1) when header, the involved segments deserve keep closer to the notary axis in compliance with the moment of momentum, in this way the players can finish the header better; and (2) during side header with both feet taking off from ground, the main factors influencing the hitting power of side header include linear and angular velocities of head, angular acceleration of posterior coxa and neck, and .hitting power has lower relative velocity with angular acceleration of head. Due to the characteristics of joint movements, the angular acceleration of both knees was rather lower.
\end{abstract}

\section{Introduction}

Header is a technique that use in association football to control the ball using the head to pass, shoot or clear. This can be done by standing, jumping or diving position. Header is a common technique and players are used in almost every match. In general, Forward used header to score a goal while defenders usually used to avoid the scoring a goal by opponent. Header is the only option when ball is in air under the rule player can't make contact with ball using hand. Most header goals scored as a result of a cross or a corner. Playmaker passes the ball across the goal in the air, and the attacking player (either standing jumping or diving position) strikes the ball with his head. In recent years footballers such as Fernando Torres, Cristiano Ronaldo, Gareth Bale, Javier Hernandez, Sergio Ramos are rated as who provide such an excellent headers in world football arena.

Some researchers performed different studies on header using several approaches. For example, Kristensen et al. used a biomechanical model to analyze the jumping header in simulated competition to give a clear picture of an optimized header.[1] Gutierrez et al. investigated the relationship between neck strength, impact, and neurocognitive function in an acute bout of soccer heading in a sample of female high school varsity soccer players.[2] Tierney et al. determined the effect of sex and soccer headgear on head impact kinematics and dynamic stabilization duringsoccer heading.[3] Haran et al. intended to determine if an acute bout of soccer heading alters postural control and pronounced self-reported symptoms of cerebral concussion.[4] Bauer et al. performed three soccer header types, namely, shooting, clearing and passing and two heading approaches, namely ,standing and jumping, were manipulated to quantify impact forces and neck muscle activity in elite female soccer players.[5] Stephens et al. presented a preliminary exploratory analysis of data collected to examine whether cumulative incidence of mild head injury, or cumulative heading frequency, are related to neuropsychological functioning in male adolescent footballers. The findings indicated the absence of neuropsychological impairment arising due to cumulative mild head injury incidence, or cumulative heading.[6] Webbe \& Ochs investigated the role of heading recency interacting with heading frequency in determining neuropsychological deficits associated with heading the ball during soccer play.[7] Schneider \& Klaus simulated head impacts to estimate the injury risk associated with soccer heading. The simulations indicated an unacceptable injury risk caused by angular head accelerations for frontal and lateral impacts at relatively low impact velocities for children, and at medium range impact velocities for adults.[8] Kapidžić et al. established which applied kinematic variables significantly contributed to the efficiency of the instep kick motion in soccer. The finding revealed that two variables significantly contributed to the speed of the ball: speed of the foot of the kicking leg at the time of contact with the ball and the distance between the 
angle support leg and center of the ball.[9] Bjelica attempted to gain relevant knowledge about important differences with respect to compare accuracy of instep kicking between preferred and non-preferred leg depending on the different intensity in a resting state, and in a state of fatigue. The findings clearly demonstrated that the young football players have much more uniformed legs at the expense of accuracy when fatigued and kick the ball with the maximal intensity.[10]

In this study, the author explored the side header using biomechanical method and hope that this study can provided some benefit suggestion on players and coaches.

\section{Stage division of header technique}

Side header with both feet taking off from ground is a complete and complicated movement which consists of various simple basic movements. To separate them helps analyze and study the complete movement. This paper, with reference to many books, divides side header with both feet taking off from ground into 6 stages based on working patterns of muscles and joint motion characteristics in actual test.

a. Squatting stage

buffer for take-off.

b. Take-off stage:

knee joints start abducting until both feet off the ground.

c. Backward abduction during soaring stage

From both feet being off the ground to the maximum backward abduction of coxa

d. Heading ball stage

From coxa flexion to head touching ball.

e. Falling staging

From head touching ball to both feet being on the ground.

f. Buffering

Movement after falling to the ground and following action.

\section{Header analysis from the perspective of moment of momentum}

From the exercise principle of multi-joint muscles, when players throw their bodies into the air and push off our chests, the force of muscular motion can save energy and make up the force shortage of multi-joint muscles when they hit balls by head. The head kicking is tough to do. The body is unsupported and squat jumping has consumed some strength. What is more, the speed of jumping in vertical direction has been about or nearly zero. When pushing off chest and stretching backbone backward to the right angle, the body and the ball move relative to one another. When the player makes movement and bend the back, the body can be regarded as a rigid body: $\Sigma \mathrm{M} \triangle \mathrm{t}=0$ when players start jumping, they stretch the chest (instead of stretch the belly) to keep the balance. Players in the air coordinate upper body, legs and trunk that the moment of momentum of legs stretching backward against the hip joint axis equals that created by upper body, though they are in opposite directions. If moment of momentum by upper body is $I_{1} \omega_{1}$, and that of legs is $I_{2} \omega_{2}$, then $I_{1} \omega_{1}+I_{2} \omega$ ${ }_{2}=0$. In the process, when the back bends to a certain radiant, muscles in the abdomen start to squeeze, and led by the trunk, the body swirls against the hip joint axis. The swirling is resulted from the function of muscles' moment of pull, the product of the integration of resultant force and the distance from back joint to resultant force.

As to individuals' segments, the moment of muscle force is outer force moment. When moment of muscle force is bigger than resisting moment, segments do controlled work; when moment of muscle force is smaller than resisting moment, segments do deformable work; when moment of muscle force equals resisting moment, segments keep balance and do static work. In the process of squats, lunges and head-kicking, segments do deformable work at the beginning; until the back bend to the utmost, the segment changes to do static work and then controlled work after it. The whole process follows the moment of momentum theorem: $\mathrm{Mdt}=\mathrm{I}_{2} \omega_{2}-\mathrm{I}_{1} \omega_{1}$, among it, $\mathrm{M}$ is the moment of variable force; 
I is the rotary inertia from head to hip joint; $\omega_{1}, \omega_{2}$ are rotational speed at different moment in the process. Thus, if we want to speed up the rotation of head, we could increase angular velocity. The theorem above shows that we have two approaches to accelerate rotational speed: one is to increase the moment of muscle $M$ from the rotation theorem ( $M=I \beta, \beta$ is angular acceleration) and moment of momentum theorem, we can see that rise of pull moment $\mathrm{M}$ for muscles against segments would lead to the increase of angular velocity or angular acceleration of segments against related joints. At the meanwhile, the moment of muscles is equal to the product of muscle contractility and arm of muscle force, that is $\mathrm{M}=\mathrm{Fr}$. if we want to enlarge the moment of muscles, then we could: Firstly, increase the muscles contraction force. This can be achieved by exercising various dynamic muscles; secondly, increase the arm of muscle force. This can be influenced by the angles of muscles in different state of tensions. More uplifting the muscles are the bigger angle of muscles' tension can be. This is because the deep muscles provide muscles located in superficial layer with bigger arm of muscle force; besides, the radian of back-bending also influences arm of muscle force. In a certain range, the larger the radian is, the distance between axis of rotation and the axis of contracting directions is further. Thus, in daily training, we should emphasize stability of body in the air as well as degree of stretch (in other words, the degree of back bending). It helps to increase the arm of muscle force. It also increase muscle force to some degree, considering the fact that tighter the muscles are (longer the initial length of contraction tension is), the bigger the contraction force is. Secondly, we could decrease rotatory inertia. The rotation theorem and the moment of momentum theorem prove that when the moment of muscle force is fixed, the rotary inertia of segment or segments against hip joint axis decreases, and the angular velocity or angular acceleration would increase without doubt. Moreover, rotational inertial of objects on a certain axis of rotation is equal to the sum which is the product of quality of each part and the square of the distance between the part to the rotational axis, which is shown as $\mathrm{I}=\Sigma \mathrm{m}_{\mathrm{i}} \mathrm{r}_{\mathrm{i}}{ }^{2}$. That explains why involved segment or segments keep close to the rotation axis. Under the guidance of this theorem, the bending of knee in squat jumping and in the motionless state in the air makes some sense.

\section{Side header from kinetic analysis}

Heading by take-off is a process that the human body applies force to the ball during soaring where external force is not available. Therefore, besides the horizontal velocity from take-off, the player has no other way but to increase the force on the ball by turning body parts based on muscle' s work.. And the angular acceleration of each part exactly reflects the exertion of each part.

We divide coxa movements into anterior coxae movements and posterior coxae movements. What influences the hitting power on ball during side header with both feet taking off from ground includes angular and linear velocities of the head, angular acceleration of coxa at the back of lumbus and neck. Judging from these data, the main factors influencing the hitting power on the ball during side header are the same with those during frontal header. However, the angular acceleration of the coxa at the back of lumbus and backward abduction angle have higher relativity than that of the coxa at the front of lumbus and backward abduction angle, which indicates that both the power and angle of the coxa lateroflexion and convolution at the back of lumbus are higher than those of the coxa at the front of lumbus. Therefore, the contraction rate of front lumbus and thigh muscles is more important than that of the back lumbus.

Generally speaking, "nodding” is required during heading to increase the player' s power of heading. But judging from the data, such statement is not precise. Players mainly rely on the angular acceleration of the neck, i.e. the line between the central point of both shoulders and that of the head, instead of the angular acceleration of head, i.e. the line between the central point of the head and the top. From the technical point of view, when players jump to head the ball, they look squarely at the ball. So they will not "nod" quickly to increase the power of heading. The back abduction angle of both knees has low relativity, which indicates that the range of activity of knees during side header after soaring is limited. 


\section{Conclusion}

When header, the involved segments deserve keep closer to the notary axis in compliance with the moment of momentum, in this way the players can finish the header better.

During side header with both feet taking off from ground, the main factors influencing the hitting power of side header include linear and angular velocities of head, angular acceleration of posterior coxa and neck. Hitting power has low relativity with angular acceleration of head. Due to the characteristics of joint movements, the angular acceleration of both knees is rather low.

\section{Acknowledgments}

This study was supported by the Sports Medicine key laboratory of General Administration of Sport of China/Sports Medicine key laboratory of Sichuan province Foundation (No:2015CTYY001) .

\section{References}

[1] Kristensen, L. B.; Andersen, T. Bull; Sørensen, H.,Optimizing Segmental Movement in the Jumping Header in Soccer. Sports Biomechanics Jul2004, Vol. 3 Issue 2, p195

[2] Gutierrez, Gregory M.; Conte, Catherine; Lightbourne, Kristian,The Relationship Between Impact Force, Neck Strength, and Neurocognitive Performance in Soccer Heading in Adolescent Females. Pediatric Exercise Science Feb2014, Vol. 26 Issue 1, p33

[3] Tierney, Ryan T.; Higgins, Michael; Caswell, Shane V.; Brady, Jessica; McHard, Krista; Driban, Jeffrey B.; Darvish, Kurosh.Sex Differences in Head Acceleration During Heading While Wearing Soccer Headgear. Journal of Athletic Training Nov/Dec2008, Vol. 43 Issue 6, p578

[4] Haran, F. J.; Tierney, R.; Wright, W. G.; Keshner, E.; Silter, M., Acute Changes in Postural Control after Soccer Heading. International Journal of Sports Medicine Apr 2013, Vol. 34 Issue 4, p350

[5] Bauer, Jeffrey A.; Thomas, Tom S.; Cauraugh, James H.; Kaminski, Thomas W.; Hass, Chris J.,Impact forces and neck muscle activity in heading by collegiate female soccer players. Journal of Sports Sciences Mar2001, Vol. 19 Issue 3, p171

[6] Stephens, Richard; Rutherford, Andrew; Potter, Douglas; Fernie, Gordon. Neuropsychological impairment as a consequence of football (soccer) play and football heading: A preliminary analysis and report on school students (13-16 years). Child Neuropsychology. Dec2005, Vol. 11 Issue 6, p513-526.

[7] Webbe, Frank M.; Ochs, Shelley R. Recency and Frequency of Soccer Heading Interact to Decrease Neurocognitive Performance. Applied Neuropsychology. 2003, Vol. 10 Issue 1, p31-41.

[8] Schneider, Klaus; Zernicke, Ronald F., Computer Simulation of Head Impact: Estimation of Head-Injury Risk During Soccer Heading. International Journal of Sport Biomechanics Nov1988, Vol. 4 Issue 4, p358

[9] Kapidžić, Alen; Huremović, Tarik; Biberovic, Alija, Kinematic Analysis of the Instep Kick in Youth Soccer Players. Journal of Human Kinetics Oct2014, Vol. 42 Issue 1, p81

[10] Bjelica, Duško; Popović, Stevo; Petković, Jovica, Comparison of Instep Kicking Between Preferred and Non-Preferred Leg in Young Football Players. Montenegrin Journal of Sports Science \& Medicine Mar 2013, Vol. 2 Issue 1, p5 